\title{
Construcción y validación de escala de calidad de servicios de salud en El Salvador
}

\author{
The creation and validation of a scale to measure the quality \\ of health services in El Salvador
}

\section{ISSN 2071-8748 \\ E-ISSN 2218-3345 \\ (c) (1) (2) \\ BY NC SA}

DOI: https://doi.org/10.5377/entorno.v0i68.8445

URI: http://hdl.handle.net/11298/1125

\section{Resumen}

El presente estudio tiene como objetivo la construcción y validación de una escala de medición de los servicios de salud en El Salvador, para ello se seleccionó una muestra no probabilística de 463 personas de ambo sexo. El tipo de estudio es instrumental con un diseño retrospectivo. La técnica de recolección de datos fue la encuesta. La media de edad total es de 35.73 años. La media de edad en hombres es de 34.73 años y en las mujeres de 36.23 años; con referencia al sexo, se tiene que 297 (64.3\%)
José Ricardo Gutiérrez-Quintanilla'

Marlon Elías Lobos-Rivera² Universidad Tecnológica de El Salvador ORCID: 0000-0003-3856-1527

Recibido: 3 de febrero de 2019 Aprobado: 4 de septiembre de 2019

1 José Ricardo Gutiérrez-Quintanilla. Es Doctor en Psicología. Profesor e investigador. Universidad Tecnológica de El Salvador. jose.gutierrez@utec.edu.sv

2 Marlon Elías Lobos-Rivera. Es Licenciado en Psicología, egresado de Maestría en Educación. Profesor e investigador. Universidad Tecnológica de El Salvador. marlon.lobos@mail.utec.edu.sv 
son mujeres y 165 (35.7\%) son hombres. Los resultados indican que se tiene una prueba con adecuada validez de contenido, validez de constructo y confiabilidad.

\section{Palabras clave}

Indicadores de salud - El Salvador - mediciones. Servicios de salud - El Salvador - mediciones. Centros médicos- El Salvador - mediciones.

\section{Introducción}

El concepto de calidad nace en el campo de la industria y las empresas, tiene su desarrollo y máxima expresión durante el siglo XX. La evaluación de los servicios de salud inicia en los Estados Unidos (EE.UU.), en la década de los 50, con la acreditación de calidad de atención en los hospitales. En este contexto, surgen muchas propuestas en algunos estados, por ejemplo, las de Chicago, Washington y la Escuela de Salud Pública de Illinois, todas centradas en la construcción de estándares para evaluar la calidad de los servicios de salud. De esta forma, aparece el Proyecto Nacional de Medición de Estándares y Desempeño de la Salud Pública de EE.UU. (Oliver, 2005), el cual establece cuatro niveles para medir la calidad de atención en salud pública: a) cumplimiento entre el 75 y el $100 \%$ ) = Sí; b) cumplimiento entre el 51 y el $75 \%=$ parcialmente alto; c) cumplimiento entre el 26 y el $50 \%$ = parcialmente bajo; y d) cumplimento $<25 \%$ ) $=\mathrm{No}$, facilitando herramientas normalizadas para medir la calidad de la atención en salud pública. En América Latina, es a partir de los años 70 que surgen algunos esfuerzos, especialmente en Brasil, Argentina, Colombia y México. En algunos de estos países se presentan mejoras importantes, mientras que en otros existe moderado desarrollo de la calidad de los servicios de salud.

En la década de los 60, Donabedian (1966) establece los principios de la calidad que debían aplicarse en los servicios sanitarios. Los aportes sobre calidad los organizó en los siguientes constructos o dimensiones: estructura (institución), el proceso y los resultados, posteriormente se incluye accesibilidad. Donabedian $(1974,1988)$ define la calidad de la atención de la salud como "proporcionar al paciente el máximo y más completo bienestar, después de haber considerado el balance de las ganancias y pérdidas esperadas, que acompañan el proceso de atención en todas
$(35,7 \%)$ men. The results show that the test has adequate content validity, construct validity and reliability.

\section{Keywords}

Health indicators - El Salvador - measurements. Health services - El Salvador - measurements. Medical centers - El Salvador - measurements.

sus partes". Esta calidad recoge el pensamiento técnico de quienes la aplican (médicos, enfermeras, administradores) con su cosmovisión y formación académica (Donabedian, 1984). Existe consenso entre los especialistas en que Donabedian es el referente obligado para tratar, evaluar y estudiar la calidad de la atención en los servicios de salud, debido a que es el autor que más ha investigado y escrito sobre el tema. El autor platea que evaluar la calidad de los servicios de salud significa hacerse desde las dimensiones de estructura (organización), proceso, resultado y accesibilidad.

La calidad de atención en los servicios de salud puede analizarse desde sus dimensiones básicas: estructura, proceso, resultados y accesibilidad. La estructura (infraestructura) es definida como el conjunto de los recursos involucrados en la producción de los servicios de salud. En la estructura se incluyen los aspectos físicos, como infraestructura física, instalaciones adecuadas, equipo médico e insumos y materiales, mobiliario; el personal de salud competente, calificado y capacitado; con reglas claras, manuales actualizados, estándares y protocolos de atención, y recursos financieros. El proceso representa la secuencia organizada y continua de distintas actividades involucradas en la producción de la atención médica. Los resultados son los productos finales del proceso de atención en los servicios de salud. Estos pueden ser reconocidos por la reducción de las tasas de mortalidad o en el aumento de la sobrevivencia, la reducción de la morbilidad, la efectividad en la evaluación diagnóstica y en el tratamiento de la enfermedad, la pronta recuperación y egreso de los pacientes (Miller-Franco et al. [QAP], 2004).

Los estudios o evaluación de la calidad en los sistemas de salud tienen tres enfoques: el primero, es el sistema de garantía de calidad desde la perspectiva institucional, el segundo, es la perspectiva de los prestadores de servicios 
de salud; y el tercero, es desde la perspectiva del usuario de los servicios de salud (Miller-Franco et al. [QAP], 2004). El primero, es un modelo de garantía de calidad, organizado y ejecutado por las instituciones de salud, como el Ministerio de Salud (Minsal). El segundo, es el sistema de garantía de calidad desde los prestadores de servicios de salud, este estaría conformado por la garantía de calidad (GC), instalado por los hospitales, clínicas, centros de salud, profesionales independientes, servicios de transporte, fundaciones e instituciones de salud no estatales, con su propio sistema de monitoreo y evaluación de calidad de los servicios de salud. La tercera, es la perspectiva de la calidad de los servicios de salud, desde la percepción del usuario, que deriva en la satisfacción de los servicios de salud recibidos. Este último es un abordaje que consiste en evaluar y analizar el grado de aceptación o satisfacción de los usuarios o pacientes de los servicios de salud recibidos en el centro que visitan. Los tres enfoques de GC tienen como propósito la calidad de los servicios de salud y la satisfacción del usuario.

Según el Minsal (2017), las dimensiones que se deben estudiar en la evaluación de la calidad de los servicios de salud son: el desempeño profesional o competencia técnica, la oportunidad, rapidez y acceso a los servicios sanitarios; la eficacia, eficiencia en el manejo administrativo, el trato y las relaciones interpersonales, la continuidad del manejo y los beneficios, la seguridad técnica, comodidad y el confort del usuario del sistema de salud.

La satisfacción del usuario es uno de los aspectos que, en términos de evaluación de los servicios de salud y calidad de atención, ha venido cobrando mayor atención en salud pública (Schneider \& Palmer, 2002), siendo considerada desde hace poco más de una década uno de los ejes de evaluación de los servicios de salud (Mira \& Aranaz, 2000). En sistemas de salud de países desarrollados (Reino Unido, Canadá, EE.UU., España) y en algunos en desarrollo (Brasil, México, Chile, Nueva Zelanda, entre otros) existen políticas sanitarias que impulsan el desarrollo de un sistema de información gerencial, que se inicia con la recogida periódica de opiniones de los usuarios sobre los servicios de salud.

El estudio fue motivado por la necesidad expresada por algunos prestadores de servicios de salud y por la observación del problema en el acceso y la calidad de los servicios de salud que frecuentemente expresa la población salvadoreña. En la primera fase del estudio se tuvo el objetivo de construir, diseñar y validar una escala de medición de la calidad de los servicios de salud desde la perspectiva del usuario. Ya que no existen instrumentos válidos y confiables para medir los constructos de la calidad de los servicios de salud, y que en la segunda fase del estudio se debía medir la calidad de los servicios de salud en El Salvador. En la construcción de la escala, se operativisaron las dimensiones propuestas por Donobedian, el Minsal y del Proyecto de garantía de calidad de la salud.

\section{Método}

\section{Participantes}

En el presente estudio, de tipo instrumental (Montero \& León, 2007), se aplicó un diseño retrospectivo, se utilizó un muestreo no probabilístico de tipo intencionado en personas que hayan asistido en un periodo de 6 meses a un centro de atención de salud. La muestra fue de 463 personas. La técnica de recolección de datos fue la encuesta. La media de edad total es de 35.73 años, con $D T$ de 13.39. En los hombres, la media de edad es de 34.73 años, con $D T$ de 13.31 , y en las mujeres, de 36.23 años, con DT de 13.40. Referente a las variables sociodemográficas, 297 (64.3\%) son mujeres y 165 (35.7\%) son hombres. Otras variables sociodemográficas, como tipo de centro de salud (institución) a la que ha asistido, residencia, rangos de edad, estado civil, entre otras, pueden observarse en la tabla 1. 
Tabla 1.

Características sociodemográficas de la muestra del estudio

\begin{tabular}{|c|c|c|}
\hline & VARIABLES & $n=463$ \\
\hline \multicolumn{3}{|c|}{ Institución } \\
\hline & Hospital público & $74(16,0 \%)$ \\
\hline & Unidades de salud & $94(20,3 \%)$ \\
\hline & ISSS & $207(44,8 \%)$ \\
\hline & Clínicas/Hospital privado & $87(18,8 \%)$ \\
\hline & Total & $462(100 \%)$ \\
\hline \multicolumn{3}{|c|}{ Género } \\
\hline & Mujeres & $297(64.3 \%)$ \\
\hline & Hombres & $165(35.7 \%)$ \\
\hline & Total & $462(100 \%)$ \\
\hline \multicolumn{3}{|c|}{ Residencia } \\
\hline & Urbana & $354(78,0 \%)$ \\
\hline & Rural & $100(22,0 \%)$ \\
\hline & Total & $454(100 \%)$ \\
\hline \multicolumn{3}{|c|}{ Edad (años) } \\
\hline & De 18 a 30 & $214(46,3 \%)$ \\
\hline & De 31 a 45 & $135(29,2 \%)$ \\
\hline & Mayores de 46 & $113(24,5 \%)$ \\
\hline & Total & $462(100 \%)$ \\
\hline \multicolumn{3}{|c|}{ Estado civil } \\
\hline & Casados/as & $148(32,0 \%)$ \\
\hline & Acompañados/as & $79(17,1 \%)$ \\
\hline & Solteros/as & $202(43,7 \%)$ \\
\hline & Divorciados/Viudos/as & $33(7,1 \%)$ \\
\hline & Total & $462(100 \%)$ \\
\hline \multicolumn{3}{|c|}{ Costos de los servicios de salud } \\
\hline & Baratos/Regulares & $91(20,0 \%)$ \\
\hline & Caros/Muy caros & $364(80,0 \%)$ \\
\hline Total & & $455(100 \%)$ \\
\hline
\end{tabular}

\section{Instrumento}

Escala de la calidad de atención de los servicios de salud (ECAS). El instrumento se construyó tras la revisión de Ios postulados teóricos (Donabedian, 1966; Miller-Franco et al. [QAP], 2004; Minsal, 2017) de varios autores e instituciones especializadas en la evaluación de la calidad de los servicios de salud. Inicialmente se seleccionó un conjunto de dimensiones o componentes que representan la calidad de los servicios de salud. Entre estas dimensiones se tienen: estructura (organización), proceso, resultado, accesibilidad/oportunidad, competencias profesionales, eficacia, eficiencia, continuidad del manejo y satisfacción del usuario. Sin embargo, tras el análisis detallado de las definiciones de estos componentes, se consideró pertinente construir los indicadores de aquellas dimensiones que mejor representan la calidad de los servicios de salud. En el segundo grupo de dimensiones, que se sistematizó en reactivos o ítems que hipotéticamente miden adecuadamente la calidad de atención de los servicios de salud desde la 
perspectiva del usuario, se tienen: estructura, proceso, resultado, accesibilidad/oportunidad, competencias profesionales y continuidad del manejo. Aunque las últimas dos dimensiones algunos autores las incluyen en la primera y segunda dimensión. Pero serán los análisis estadísticos, mediante el análisis factorial de componentes principales, los que configuren las dimensiones según el patrón de respuestas expresadas por los participantes en el estudio.

En la propuesta inicial del instrumento, se construyó un universo de 51 reactivos o preguntas, pensados y organizados teóricamente para medir las dimensiones o subescalas: estructura (ítems del 1 al 12), proceso-resultado (ítems del 13 al 28), accesibilidad/oportunidad (ítems del 29 al 36), competencias profesionales (ítems 37 al 46), y continuidad del manejo (ítems del 47 al 51). Después de administrar el universo de reactivos a una muestra de 463 personas de ambos sexos y mayores de edad. fueron procesados los datos en el paquete estadístico SPSS versión 22.0. Seguidamente se iniciaron los análisis psicométricos de la prueba.

\section{Resultados}

Tras la construcción de la muestra de 51 reactivos con una escala de medida tipo Likert, donde 1= mala/o, 2= regular, $3=$ buena/o y $4=$ muy buena/o, se solicitó a cinco profesionales especializados en el tema que revisaran la redacción de los ítems y la pertinencia de estos para medir las dimensiones propuestas, dando como resultado que la mayoría coincidió en que los ítems estaban construidos adecuadamente para medir las dimensiones propuestas. En consecuencia, se puede afirmar que el instrumento en construcción posee adecuados criterios de validez de contenido para medir la calidad de atención de los servicios de salud en El Salvador.
La validez de constructo. En la primera fase de este proceso se realizó un análisis factorial mediante el método de componentes principales. Tanto el análisis Káiser-MeyerOlkin (KMO $=.97)$ como la prueba de esfericidad de Bartlett $(X 2(463)=14413,61 ; p=.001)$ indicaron la adecuación de los datos para este tipo de análisis. Siguiendo el procedimiento de factores con rotación varimax, resultó en una solución de cinco factores que explican el $60,46 \%$ de la varianza total explicada, presentando todos los ítems valores de saturación superiores a .40; a excepción del reactivo 48 (el cumplimiento de las citas por parte del centro de salud fue) que tuvo una carga factorial inferior a .40, por lo que fue excluido de la prueba. En la tabla 2 se pueden ver los análisis anteriores.

El análisis factorial mediante componentes principales, modificó la propuesta hipotética original de los factores en una solución siempre de cinco factores, pero con moderadas modificaciones. Los nuevos factores son: proceso-resultado, estructura (organización), actitud diligente, accesibilidad/oportunidad y distancia del centro de salud. La dimensión satisfacción del usuario resulta de la sumatoria o puntaje total de los ítems de la prueba. En este sentido, se describe cada una de las dimensiones que resultaron del análisis factorial de la escala: el factor uno, proceso-resultado, conformado con quince ítems $(15,17$, $18,20,21,22,25,26,28,37,38,40,45,46,50)$; el factor dos, estructura, integrado con trece ítems $(1,2,3,4,5,6$, $7,8,9,10,11,12,49)$; el factor tres, actitud diligente, lo conforman nueve ítems (16, 23, 24, 27, 39, 41, 42, 43, 44); el factor cuatro, accesibilidad/oportunidad, lo conforman diez ítems $(13,14,19,32,3,34,35,36,47,51)$; y el factor cinco, distancia del centro de salud, lo conforman tres ítems $(29,30,31)$. 
Tabla 2.

Matriz de componentes principales (factores), su carga factorial rotada y el porcentaje de varianza total explicada por cada dimensión de la escala de calidad de atención de los servicios de salud

\begin{tabular}{|c|c|c|c|c|c|}
\hline \multirow[b]{2}{*}{ Reactivos } & \multicolumn{5}{|c|}{ Factores/Componentes } \\
\hline & $\begin{array}{l}\text { Proceso- } \\
\text { resultado } \\
\text { (Factor 1) }\end{array}$ & $\begin{array}{l}\text { Estructura } \\
\text { (Factor 2) }\end{array}$ & $\begin{array}{c}\text { Actitud } \\
\text { diligente } \\
\text { (Factor 3) }\end{array}$ & $\begin{array}{c}\text { Accesibilidad / } \\
\text { Oportunidad } \\
\text { (Factor 4) }\end{array}$ & $\begin{array}{l}\text { Distancia del centro } \\
\text { de salud (Factor 5) }\end{array}$ \\
\hline A25 & .712 & & & & \\
\hline A21 & .706 & & & & \\
\hline A26 & .702 & & & & \\
\hline A20 & .700 & & & & \\
\hline A45 & .675 & & & & \\
\hline A46 & .629 & & .403 & & \\
\hline A28 & .628 & & & & \\
\hline $\mathrm{A} 40$ & .624 & & & & \\
\hline A50 & .622 & & & & \\
\hline $\mathrm{A} 17$ & .620 & & & & \\
\hline A18 & .584 & .408 & & & \\
\hline A38 & .578 & & & & \\
\hline A22 & .576 & & & & \\
\hline A15 & .514 & & & & \\
\hline A37 & .422 & & .404 & & \\
\hline $\mathrm{A} 1$ & & .717 & & & \\
\hline A5 & & .712 & & & \\
\hline A4 & & .695 & & & \\
\hline $\mathrm{A} 2$ & & .679 & & & \\
\hline A7 & & .659 & & & \\
\hline A6 & & .630 & & & \\
\hline A10 & & .616 & & & \\
\hline A8 & & .613 & & & \\
\hline A3 & & .602 & & & \\
\hline A11 & & .557 & & & \\
\hline A9 & & .529 & & & \\
\hline A19 & & .457 & & .447 & \\
\hline $\mathrm{A} 12$ & & .447 & & .400 & \\
\hline A49 & & .438 & .404 & & \\
\hline A43 & & & .730 & & \\
\hline A44 & & & .700 & & \\
\hline A42 & & & .691 & & \\
\hline A41 & & & .633 & & \\
\hline A27 & & & .633 & & \\
\hline
\end{tabular}




\begin{tabular}{|c|c|c|c|c|c|}
\hline A39 & & & .617 & & \\
\hline A24 & & & .601 & & \\
\hline $\mathrm{A} 16$ & & .426 & .534 & & \\
\hline A23 & & & .467 & & \\
\hline A32 & & & & .572 & \\
\hline A33 & & & .452 & .516 & \\
\hline A14 & & .402 & & .506 & \\
\hline $\mathrm{A} 13$ & & .413 & & .499 & \\
\hline A34 & & & & .487 & \\
\hline A36 & & & & .475 & \\
\hline A47 & .403 & & & .473 & \\
\hline A35 & & & & .438 & \\
\hline A51 & .408 & & & .426 & \\
\hline A31 & & & & & .804 \\
\hline A 29 & & & & & .776 \\
\hline $\mathrm{A} 30$ & & & & &, 580 \\
\hline$\%$ Varianza & & & & & \\
\hline $\begin{array}{c}\text { total } \\
\text { explicada }\end{array}$ & 46,49 & 4,57 & 3,92 & 3,16 & 2,31 \\
\hline$a$ & .95 & .92 & .92 & .90 & .76 \\
\hline
\end{tabular}

Análisis de confiabilidad

Tras el análisis factorial que brindó una configuración de la prueba en cinco componentes, se procedió al análisis de consistencia interna mediante el método de Alfa de Cronbach. El propósito de este análisis de ítems y de consistencia interna es la obtención de índices o criterios empíricos de confiabilidad de los cinco factores obtenidos mediante el análisis de componentes principales, en el cual se incluye la estructura factorial y los ítems que conforman cada subescala.

En el factor (subescala) proceso-resultado. Se tuvo un valor de consistencia interna alfa de Cronbach de .95. La media de respuesta a los ítems osciló entre 2.46 (ítem 15) y 2.82 (ítem 38). En casi todos los ítems la media se encuentra por arriba de la media de la escala, que es de 2.5. Las desviaciones estándar en la mayoría de los ítems están cercanas a la unidad; la correlación ítems-total de los reactivos son superiores a .60. Tal como se observa en la tabla 4, junto a estos estadísticos se calculó la correlación ítems-total, corregida, y el alfa de Cronbach si se elimina el ítem. En esta subescala, se agruparon los ítems de competencia técnica o profesional que (como hipótesis) inicial se habían redactado como una dimensión independiente. 
Tabla 4.

Media (M), desviación estándar (DE), correlación ítems-total (Ri-T) y el Alfa de Cronbach si el ítem es eliminado $(\alpha)$ en la dimensión proceso-resultado

\begin{tabular}{ccccc}
\hline Reactivos & $M$ & $D E$ & Ri-T & $a$ \\
\hline A15 & 2.46 & 0.87 & .687 & .950 \\
A17 & 2.65 & 0.82 & .757 & .948 \\
A18 & 2.63 & 0.90 & .745 & .949 \\
A20 & 2.70 & 0.81 & .737 & .949 \\
A21 & 2.73 & 0.81 & .743 & .949 \\
A22 & 2.61 & 0.80 & .753 & .949 \\
A25 & 2.65 & 0.87 & .765 & .948 \\
A26 & 2.65 & 0.85 & .776 & .948 \\
A28 & 2.68 & 0.77 & .751 & .949 \\
A37 & 2.53 & 0.78 & .644 & .951 \\
A38 & 2.82 & 0.75 & .679 & .950 \\
A40 & 2.70 & 0.78 & .683 & .950 \\
A45 & 2.64 & 0.84 & .803 & .947 \\
A46 & 2.60 & 0.87 & .761 & .948 \\
A50 & 2.69 & 0.82 & .752 & .949 \\
\hline
\end{tabular}

En la subescala estructura/organización. obtuvo un valor de consistencia interna alfa de Cronbach de .92. La media de respuesta a los ítems osciló entre 2.30 (ítem 7) y 2.65 (ítem 4). La mayoría de los ítems ronda la media de la escala (2.5), aunque en algunos (cuatro) sus medias son superiores a la media de la escala. Las desviaciones estándar en la mayoría de los ítems están cercanas a la unidad. La correlación ítemstotal de los reactivos es superior a .50. Tal como se observa en la tabla 5, junto a estos estadísticos se calculó la correlación ítems-total, corregida, y el alfa de Cronbach si el ítem es eliminado.

Tabla 5.

Media (M), desviación estándar (DE), correlación ítems-total (Ri-T) y el alfa de Cronbach si el ítem es eliminado $(\alpha)$ en la dimensión estructura/institución

\begin{tabular}{ccccc}
\hline Reactivos & $M$ & $D E$ & Ri-T & A \\
\hline A1 & 2.53 & 0.85 & .666 & .923 \\
A2 & 2.47 & 0.80 & .666 & .923 \\
A3 & 2.41 & 0.83 & .691 & .923 \\
A4 & 2.65 & 0.91 & .676 & .923 \\
A5 & 2.50 & 0.83 & .709 & .922
\end{tabular}




$\begin{array}{lllll}\text { A6 } & 2.48 & 0.83 & .703 & .922 \\ \text { A7 } & 2.30 & 0.89 & .745 & .921 \\ \text { A8 } & 2.30 & 0.86 & .725 & .921 \\ \text { A9 } & 2.41 & 0.84 & .628 & .925 \\ \text { A10 } & 2.55 & 0.87 & .711 & .922 \\ \text { A11 } & 2.46 & 0.88 & .676 & .923 \\ \text { A12 } & 2.46 & 0.93 & .567 & .927 \\ \text { A49 } & 2.41 & 0.82 & .683 & .923\end{array}$

En la subescala actitud diligente. Se obtuvo un valor de consistencia interna alfa de Cronbach de .92. La media de respuesta a los ítems osciló entre 2.13 (ítem 30) y 2.41 (ítem 42). En todos los ítems, la media se encuentra relativamente por debajo de la media de la escala, que es de 2.5. Las desviaciones estándar en la mayoría de los ítems están cercanas a la unidad. La correlación ítems-total de los reactivos son superiores a .60. Tal como se observa en la tabla 6, junto a estos estadísticos se calculó la correlación ítems-total, corregida, y el Alfa de Cronbach si se elimina el ítem.

Tabla 6.

Media (M), desviación estándar (DE), correlación ítems-total (Ri-T) y el Alfa de Cronbach si el ítem es eliminado $(\alpha)$ en la dimensión actitud diligente

\begin{tabular}{ccccc}
\hline Reactivos & $M$ & $D E$ & Ri-T & A \\
\hline A16 & 2.30 & 0.92 & .699 & .917 \\
A23 & 2.13 & 0.84 & .600 & .923 \\
A24 & 2.35 & 0.84 & .739 & .915 \\
A27 & 2.38 & 0.92 & .688 & .918 \\
A39 & 2.33 & 0.90 & .731 & .915 \\
A41 & 2.38 & 0.90 & .730 & .915 \\
A42 & 2.41 & 0.87 & .783 & .912 \\
A43 & 2.26 & 0.90 & .814 & .910 \\
A44 & 2.31 & 0.88 & .755 & .914 \\
\hline
\end{tabular}

En la subescala accesibilidad/oportunidad. Se obtuvo un valor de consistencia interna Alfa de Cronbach de .90. La media de respuesta a los ítems osciló entre 2.02 (ítems 32,33 ) y 2.54 (ítem 51). En la mayoría de ítems, la media es inferior a la media de la escala, que es de 2.5. Las desviaciones estándar en la mayoría de los ítems están cercanas a la unidad, la correlación ítems-total de los reactivos son superiores a .60. Tal como se observa en la tabla 7, junto a estos estadísticos se calculó la correlación ítems-total, corregida, y el alfa de Cronbach si se elimina el ítem. 
Tabla 7.

Media (M), desviación estándar (DE), correlación ítems-total (Ri-T) y el alfa de Cronbach si el ítem es eliminado $(\alpha)$ en la dimensión accesibilidad/oportunidad

\begin{tabular}{ccccc}
\hline Reactivos & $M$ & $D E$ & Ri-T & a \\
\hline A13 & 2.42 & 0.92 & .611 & .896 \\
A14 & 2.47 & 0.92 & .625 & .895 \\
A19 & 2.35 & 0.93 & .677 & .892 \\
A32 & 2.02 & 0.95 & .719 & .889 \\
A33 & 2.02 & 0.93 & .676 & .892 \\
A34 & 2.21 & 0.92 & .626 & .895 \\
A35 & 2.24 & 0.86 & .662 & .893 \\
A36 & 2.11 & 0.91 & .609 & .896 \\
A47 & 2.33 & 0.88 & .664 & .893 \\
A51 & 2.54 & 0.92 & .698 & .891 \\
\hline
\end{tabular}

En la subescala distancia del centro de salud se obtuvo un valor de consistencia interna Alfa de Cronbach de .76. La media de respuesta a los ítems osciló entre 2.35 (ítem 30) y 2.64 (ítem 29). En dos de los tres ítems, la media se encuentra por arriba de la media de la escala, que es de 2.5, a excepción del ítem 30, el cual tiene una media inferior a la de la escala. Las desviaciones estándar en la mayoría de los ítems están cercanas a la unidad, la correlación ítems total de los reactivos son superiores a .60. Como se observa en la tabla 8, junto a estos estadísticos se calculó la correlación ítems-total, corregida, y el Alfa de Cronbach si se elimina el ítem.

Tabla 8.

Media (M), desviación estándar (DE), correlación ítems-total (Ri-T) y el alfa de Cronbach si el ítem es eliminado $(\alpha)$ en la dimensión distancia del centro de salud

\begin{tabular}{ccccc}
\hline Reactivos & $M$ & $D E$ & Ri-T & A \\
\hline A29 & 2.64 & 0.86 & .642 & .633 \\
A30 & 2.35 & 0.88 & .485 & .804 \\
A31 & 2.53 & 0.89 & .672 & .595 \\
\hline
\end{tabular}

Análisis inferencial

En el análisis inferencial de resultados se aplicaron pruebas paramétricas, como la prueba t de Student para muestras independientes, el análisis de varianza (Anova) y las correlaciones de Pearson. Contrastándose las variables o dimensiones de la calidad de atención de los servicios de salud o variables criterio (dependientes) en función de las variables predictoras o independientes (cualitativas), y se contrastarán por el tipo de institución, de residencia y por los costos por enfermarse y curarse. 
Para contrastar las dimensiones de la calidad de atención de los servicios de salud en función del tipo de institución, se aplicó el análisis de varianza (Anova factorial). En el análisis de la dimensión proceso-resultado se encontraron diferencias estadísticas entre los hospitales públicos, unidades de salud, Instituto Salvadoreño del Seguro Social (ISSS) y clínicas y hospitales privados $(M=36.49$, $D E=9.13 ; M=35.70, D E=8.57 ; M=35.23, D E=7.96 ; M$ $=44.49, D E=7.98) ; F(3.422)=25.38 ; p<.001 ; f=.154$. La aplicación de la prueba post hoc de Scheffé demostró que existen diferencias significativas entre las clínicas y hospitales privados con los hospitales públicos $(p<.001)$; con las unidades de salud $(p<.001)$; y con los centros de salud del ISSS $(p<.001)$, presentando mayores problemas de calidad de atención de la salud en el proceso-resultado los hospitales públicos, las unidades de salud y los centros de salud del ISSS. Indicando un efecto grande del tipo de institución de salud en el proceso-resultado de la calidad de atención de la salud.

Con respecto a la variable estructura/institución, se encontraron diferencias estadísticas significativas entre los hospitales públicos, unidades de salud, ISSS y clínicas y hospitales privados $(M=27.21, D E=7.25 ; M=28.08, D E=$ $7.06 ; M=28.75, D E=6.58 ; M=36.15, D E=7.59) ; F(3.422)=$ $28.79 ; p<.001 ; f=.166$. La aplicación de la prueba post hoc de Scheffé demostró que existen diferencias significativas entre las clínicas y hospitales privados con los hospitales públicos $(p<.001)$; con las unidades de salud $(p<.001)$; y con los centros de salud del ISSS $(p<.001)$, presentando mayores problemas de calidad de atención de la salud en la estructura/institución los hospitales públicos, las unidades de salud y los centros de salud del ISSS, indicando un efecto grande del tipo de institución de salud en la estructura/ institución de la calidad de atención de la salud. Al analizar la variable accesibilidad/oportunidad, se encontraron diferencias estadísticas entre hospitales públicos, unidades de salud, ISSS y clínicas y los hospitales privados $(M=19.70$, $D E=6.30 ; M=20.06, D E=6.07 ;[M=18.72, D E=4.86 ; M$ $=25.96, D E=5.59)] ; F(3.446)=34.58 ; p<.001 ; f=.190$. La aplicación de la prueba post hoc de Scheffé demostró que existen diferencias significativas entre las clínicas y hospitales privados con los hospitales públicos $(p<.001)$; con las unidades de salud $(p<.001)$; y con los centros de salud del ISSS $(p<.001)$, presentando mayores problemas de calidad de atención de la salud debido a la accesibilidad/ oportunidad, los hospitales públicos, las unidades de salud y Ios centros de salud del ISSS. Indicando un efecto grande del tipo de institución de salud en la accesibilidad/oportunidad de la calidad de atención de la salud

Para el análisis de las dimensiones de la calidad de atención en los centros de salud en función de los costos por enfermarse y curarse, se aplicó la prueba t de Student para muestras independientes. En el análisis del factor procesoresultado en función de los costos de los servicios de salud, se encontró que existen diferencias estadísticas significativas entre las personas que dicen que los costos son baratos/ económicos y las personas que dicen que los costos son caros o muy caros $(M=40.85, D E=8.55 ; M=36.35, D E$ $=8.89) ; t(414)=4.18 ; p<.001 ; d=.52$, presentando un efecto medio el grupo de personas que dicen que los costos son caros o muy caros en comparación con los que dicen que los costos son baratos/ económicos. En el contraste con el factor actitud diligente en función de los costos de los servicios de salud, se encontró que existen diferencias estadísticas significativas entre las personas que dicen que los costos son baratos o económicos y las personas que dicen que los costos son caros o muy caros $(M=21.50, D E=$ $5.42 ; M=18.11, D E=5.54) ; t(443)=5.17 ; p<.001 ; d=.62$, presentando un efecto medio el grupo personas que dicen que los costos son caros o muy caros, en comparación con los que dicen que los costos son baratos o económicos. En el contraste con el factor accesibilidad/oportunidad en función de los costos de los servicios de salud, se encontró que existen diferencias estadísticas significativas entre personas que dicen que los costos son baratos o económicos y las personas que dicen que los costos son caros o muy caros $(M=21.50, D E=5.42 ; M=18.11, D E=$ $5.54) ; t(439)=5.17 ; p<.001 ; d=.55 ;$ presentando un efecto medio el grupo de personas que dicen que los costos son caros o muy caros, en comparación con los que dicen que los costos son baratos.

\section{Discusión}

Existen diferentes postulados teóricos que definen y explican la evaluación de la calidad de atención de la salud. La mayoría de estos autores presentan coincidencia referente a las dimensiones que deberán medirse al evaluar la calidad de la salud (Donabedian, 1966; Miller-Franco et al. [QAP], 2004; OPS/OMS, 2007; Minsal, 2017). Sin embargo, algunos enfatizan en unos componentes mientras otros insisten en otras dimensiones. La evaluación de la calidad de los servicios de salud y la creación de indicadores estandarizados comenzó en la década de los 50 en EE.UU., 
pero estos esfuerzos iniciaron en 1910. En América Latina, los primeros esfuerzos comenzaron en la década de los 70 del siglo pasado. En El Salvador existió un primer intento infructuoso en el año 2004, pero fue hasta en el 2013 que comenzó a instalarse formalmente un sistema de GC en el sector público de la salud. La instalación de un sistema de $\mathrm{GC}$, en los establecimientos que prestan servicios de salud, también debe conllevar un proceso de evaluación de la calidad con sus indicadores y criterios de cumplimiento.

En la presente investigación, que tenía como propósito la construcción y validación de una escala de medición de la calidad de los servicios de salud en El Salvador, se siguió la propuesta de Nunnally y Bernstein, (1995), quienes expresan que, cuando se construye una prueba, el objetivo es conseguir un grupo de ítems que maximice la varianza del test, seleccionando para ello a aquellos con un elevado poder de discriminación, alta desviación típica y con puntuaciones medias de respuesta situadas en torno al punto medio de la escala.

Como resultado del estudio y de los análisis psicométricos, la prueba final quedó constituida por las dimensiones o subescalas siguientes: proceso-resultado, estructura/ institución, actitud diligente, accesibilidad/oportunidad y distancia del centro de salud. El análisis factorial mediante componentes principales fue significativo y el KMO superior a .90, la varianza total explicada superior al $60 \%$ y la carga factorial de los reactivos superior a .40 , indicando que la prueba posee una adecuada estructura factorial $y$, en consecuencia, buenas propiedades de validez de constructo. Estos resultados coinciden con los postulados de Gronlund (1976), quien plantea que la validez de constructo intenta responder las preguntas ihasta dónde un instrumento mide realmente un determinado rasgo latente 0 una característica de las personas? y ¿con cuánta eficiencia lo hace? En consecuencia, es necesario que podamos mostrar evidencia de que, efectivamente, el instrumento mide el rasgo o constructo que se pretende medir.

En la misma dirección, Carretero-Dios, y Pérez, (2005) expresan que es relevante destacar que, si un constructo está configurado por distintos factores o componentes (dimensiones), los cálculos de discriminación tienen que hacerse por factores y no considerando el total de la escala. La idea es que cada componente del constructo debe ser una categoría homogénea de contenido y "aislada", en la medida de lo posible, del resto de componentes, de lo contrario no puede sostenerse su separación como categorías distintas de un mismo constructo. En relación con los análisis de confiabilidad, se aplicó la consistencia interna alfa de Cronbach; y las cinco subescalas poseen índices superiores a .70. En todos los ítems, la correlación ítems-total es mayor a .30, indicando que la prueba posee criterios métricos de confiabilidad adecuados para medir los constructos en muestras salvadoreñas.

En la mayoría de las dimensiones de la escala de calidad de atención de los servicios de salud, al compararlas por establecimientos, se tiene que son las clínicas/hospitales privados los que obtienen un mayor puntaje que los hospitales públicos, unidades de salud y el ISSS, indicando que son los primeros centros los que prestan un mejor servicio de atención. Esto se cumple en las dimensiones: proceso-resultado, estructura/institución, actitud diligente y accesibilidad/oportunidad. Los análisis refieren que existe un efecto grande del tipo de centro de salud en la calidad de atención de la salud. Al comparar las dimensiones por residencia de la muestra, se tiene que hay diferencias en proceso-resultado y en institución y actitud diligente, presentando una inferior percepción de calidad de atención de la salud los residentes urbanos que los rurales, con un efecto pequeño. No hay diferencia entre las demás dimensiones. Con referencia a los costos de enfermarse y curarse en El Salvador, los datos indican, en las diferentes dimensiones, que las personas que opinan que curarse en nuestro país es caro o muy caro son las que hacen una peor evaluación de la calidad de los servicios de salud, comparando con las que perciben como barato y económica la salud. El efecto es medio, en relación con los costos de salud, en la calidad de los servicios de la salud. En conclusión, se puede afirmar que la prueba posee adecuadas propiedades psicométricas de validez de contenido, validez de constructo y confiabilidad para medir la calidad de los servicios de salud en El Salvador.

\section{Referencias}

Carretero-Dios, H. \& Pérez, C. (september, 2007). Standards for the development and review of instrumental studies: Considerations about test selection in psychological research. International Journal of Clinical and Health Psychology, 7(3), 863-882.

Donabedian, A. (july, 1966). Evaluating the quality of medical care. Milbank Memorial Fund Quarterly, 44(3), 166-203. 
Donabedian, A. (november, 1974). Concepts of health care quality: A perspective. Washington, D. C., United States: National Academy of Sciences, Institute of Medicine.

Donabedian, A. (marzo-abril, 1988). Veinte años de investigación en torno a la calidad de la atención médica, 1964-1984. Salud Pública Méx, 30(2), 202-215.

Donabedian, A. (may-june, 1984). Quality, cost, and cost containment. Nurs Outlook, 32(3), 142-145.

Gronlund, N. E. (1976). Elaboración de tests de aprovechamiento. México, D. F., México: Trillas.

Miller Franco, L., Silimperi, D. R., Veldhuyzen van Zanten, T., MacAulay, C., Askov, K., Bouchet, B.,... Márquez, L. (2004). Sostenibilidad de la calidad en la atención de salud: Institucionalización de la garantía de la calidad. Recuperado de https://www.usaidassist.org/ sites/default/files/sostenibilidad_de_la_calidad_de_la_ atencion_de_salud_2004.pdf

Ministerio de Salud. (2017). Dimensiones de la calidad de atención de los centros de salud. San Salvador, El Salvador.
Mira, J. J. y Aranaz, J. (2000). La satisfacción del paciente como una medida de resultado de la atención sanitaria. Medicina Clínica, 114(3), 26-33.

Montero, I. \& León, O. G. (2007). A guide for naming research studies in psychology. International Journal of Clinical and Health Psychology, 7(3), 847-862.

Nunnally, J. C. y Bernstein, I. H. (1995). Teoría psicométrica. Madrid, España: McGraw-Hill.

Oliver, J. G. (julio-agosto, 2005). Calidad en salud pública. GaC Sanit, 19(4), 325-32.

Organización Panamericana de la Salud y Organización Mundial de la Salud. (2007). Política y estrategia regional para la garantía de la calidad de la atención sanitaria, incluyendo la seguridad del paciente [Conferencia]. Recuperado de http://iris.paho.org/ xmlui/ bitstream/handle/123456789/3760/csp27. r10-s.pdf?sequence $=2 \&$ isAllowed $=y$

Schneider, H. y Palmer, N. (2002). Getting to the truth? Researching user views of primary health care. Health Policy Plan, 17(1), 32-41. 


\section{ESCALA DE CALIDAD DE ATENCIÓN DE LOS SERVICIOS DE SALUD (ECAS)}

Indicaciones: El presente estudio tiene como propósito evaluar la calidad de atención de los servicios de salud en El Salvador. Para ello, estamos solicitando su colaboración respondiendo cada una de las preguntas que se le hacen. Se pide que responda una sola vez en cada pregunta, colocando una equis $(X)$ en la casilla del número que aparecen a la derecha de cada pregunta. Favor responda de acuerdo con las vivencias, experiencias personales u observaciones en el centro de salud que regularmente asiste. La información facilitada será tratada confidencialmente. MUCHAS GRACIAS.

\section{DATOS GENERALES}

1. Institución de salud a la que regularmente asiste:
1. Hospital público
2. Unidad de salud
3. Seguro social
4. Clínica/hospital privado
5. ECOS

2. Género:

1 Femenino

2. Masculino

3. Residencia:

1. Urbana

2. Rural

4. Edad: años

5. Estado Civil:

1. Casado (a)

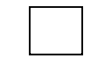

2. Acompañado(a)

3. Divorciado(a)

4. Soltero(a)

5. Viudo(a)

6. Escolaridad:

7. Número de miembros en su hogar:

8. Piensa usted que los costos de enfermarse y curarse en El Salvador son:

1. Baratos

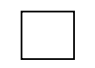

2. Económicos

3. Caros

4. Muy caros

9. Departamento:

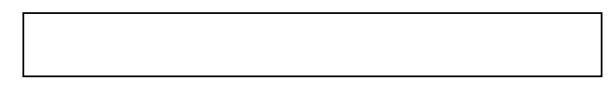




\section{Forma de responder}

$1=$ Mala(s); 2 = Regular; 3 = Buena (o); 4 = Muy buena(0)

\begin{tabular}{|c|c|c|c|c|c|}
\hline No. & Ítems & 1 & 2 & 3 & 4 \\
\hline 1 & Las instalaciones físicas (infraestructura) del centro de salud son & 1 & 2 & 3 & 4 \\
\hline 2 & La distribución física de los departamentos y unidades del centro de salud son & 1 & 2 & 3 & 4 \\
\hline 3 & La organización funcional (toma de decisiones) del centro de salud es & 1 & 2 & 3 & 4 \\
\hline 4 & El centro de salud está limpio y ordenado & 1 & 2 & 3 & 4 \\
\hline 5 & La conservación y mantenimiento del centro de salud es & 1 & 2 & 3 & 4 \\
\hline 6 & La dirección y administración del centro de salud es & 1 & 2 & 3 & 4 \\
\hline 7 & La comodidad básica del paciente cuando está ingresado en el centro de salud es & 1 & 2 & 3 & 4 \\
\hline 8 & La comodidad básica del paciente cuando tiene consulta externa es & 1 & 2 & 3 & 4 \\
\hline 9 & El equipamiento de bancas, sillas y escritorios son & 1 & 2 & 3 & 4 \\
\hline 10 & Los aparatos, equipo médico y materiales son & 1 & 2 & 3 & 4 \\
\hline 11 & El proceso de atención sigue un protocolo estándar (ordenado) & 1 & 2 & 3 & 4 \\
\hline 12 & La existencia de medicamentos e insumos médicos (jeringas, descartables) son & 1 & 2 & 3 & 4 \\
\hline 13 & La elaboración del expediente clínico y entrega de medicamentos son & 1 & 2 & 3 & 4 \\
\hline 14 & La toma de exámenes clínicos (laboratorio y rayos $\mathrm{X}$ ) y entrega de resultados son & 1 & 2 & 3 & 4 \\
\hline 15 & El seguimiento de la evolución de la enfermedad por el médico es & 1 & 2 & 3 & 4 \\
\hline 16 & La atención que recibe de la enfermera(o) es & 1 & 2 & 3 & 4 \\
\hline 17 & La evaluación diagnóstica y el tratamiento médico fue & 1 & 2 & 3 & 4 \\
\hline 18 & Observa diligencia y esfuerzo en el médico para tratar su problema de salud & 1 & 2 & 3 & 4 \\
\hline 19 & Lo administrativo como sacar una cita, solicitar una constancia médica es & 1 & 2 & 3 & 4 \\
\hline 20 & La medicina indicada por el médico fue & 1 & 2 & 3 & 4 \\
\hline 21 & El tratamiento que le indico el médico para curar la enfermedad fue & 1 & 2 & 3 & 4 \\
\hline 22 & La impresión general de la atención médica recibida fue & 1 & 2 & 3 & 4 \\
\hline 23 & La atención del sistema de salud salvadoreño (hospital, clínica, unidad de salud) es & 1 & 2 & 3 & 4 \\
\hline 24 & Los cuidados e indicaciones que recibe de las(os) profesionales de enfermería son & 1 & 2 & 3 & 4 \\
\hline 25 & La explicación que le dio el médico tratante sobre la enfermedad fue & 1 & 2 & 3 & 4 \\
\hline 26 & La explicación que le dio el médico tratante sobre el tratamiento fue & 1 & 2 & 3 & 4 \\
\hline 27 & La enfermera(o) le orienta sobre el proceso que debe cumplir con su tratamiento? & 1 & 2 & 3 & 4 \\
\hline 28 & La mejora de su salud después de la visita al centro de salud fue & 1 & 2 & 3 & 4 \\
\hline 29 & La accesibilidad por la distancia a un centro de salud es & 1 & 2 & 3 & 4 \\
\hline 30 & Los costos en dinero para asistir a una consulta médica son & 1 & 2 & 3 & 4 \\
\hline
\end{tabular}




\begin{tabular}{|c|c|c|c|c|c|}
\hline 31 & El trasladarse de su casa a un centro de salud es & 1 & 2 & 3 & 4 \\
\hline 32 & El tiempo de espera entre una cita y la otra, fue & 1 & 2 & 3 & 4 \\
\hline 33 & Al presentarse a la consulta médica el tiempo de espera fue & 1 & 2 & 3 & 4 \\
\hline 34 & Los horarios de atención médica (consulta y emergencias) en El Salvador son & 1 & 2 & 3 & 4 \\
\hline 35 & El acceso al médico general en el sistema de salud es & 1 & 2 & 3 & 4 \\
\hline 36 & El acceso al médico especialista en el sistema de salud es & 1 & 2 & 3 & 4 \\
\hline 37 & Las habilidades de los profesionales de la salud para hacer su trabajo son & 1 & 2 & 3 & 4 \\
\hline 38 & Los conocimientos que tiene el médico tratante son & 1 & 2 & 3 & 4 \\
\hline 39 & Las actitudes (disposición) del personal de salud fue & 1 & 2 & 3 & 4 \\
\hline 40 & Los criterios que posee el médico para elaborar el diagnóstico y tratamientos son & 1 & 2 & 3 & 4 \\
\hline 41 & El trato (humano) de los profesionales de la salud fue & 1 & 2 & 3 & 4 \\
\hline 42 & La diligencia y esfuerzo en la atención del personal de salud fue & 1 & 2 & 3 & 4 \\
\hline 43 & El interés y la atención de la enfermera(o) fue & 1 & 2 & 3 & 4 \\
\hline 44 & Las relaciones interpersonales del personal de enfermeras fue & 1 & 2 & 3 & 4 \\
\hline 45 & El interés que mostró el médico tratante en su problema fue & 1 & 2 & 3 & 4 \\
\hline 46 & El humanismos que mostró el médico tratante en su problema fue & 1 & 2 & 3 & 4 \\
\hline 47 & El seguimiento o continuidad entre una cita y la siguiente fue & 1 & 2 & 3 & 4 \\
\hline 48 & La permanencia o disposición de los servicios de salud en el centro son & 1 & 2 & 3 & 4 \\
\hline 49 & Percibió mejoría y beneficios en su salud después de la consulta al medico & 1 & 2 & 3 & 4 \\
\hline 50 & Los exámenes clínicos fueron indicados y realizados en un tiempo razonable & 1 & 2 & 3 & 4 \\
\hline
\end{tabular}

